\title{
Novel Therapeutics for Adverse Effects of Antitumor Therapy: The Promise of Multicomponent, Traditional Japanese Herbal Remedies
}

\author{
Toru Kono $^{1-3^{*},}$ Hiroshi Takeda ${ }^{2}$, Mitsuo Shimada ${ }^{3,}$ Yoshio Kase ${ }^{4}$ and Yasuhito Uezono ${ }^{5}$ \\ ${ }^{1}$ Center for Clinical and Biomedical Research, Sapporo Higashi Tokushukai Hospital, Sapporo, Japan \\ ${ }^{2}$ Pathophysiology and Therapeutics, Hokkaido University Faculty of Pharmaceutical Sciences, Sapporo, Japan \\ ${ }^{3}$ Department of Surgery, Institute of Health Biosciences, The University of Tokushima Graduate School of Medicine, Tokushima, Japan \\ ${ }^{4}$ Tsumura Research Laboratories, Tsumura \& Co., 3586 Yoshiwara, Ami-machi, Inashiki-gun, Japan \\ ${ }^{5}$ Division of Cancer Pathophysiology, National Cancer Center Research Institute, Tsukiji, Japan
}

"Corresponding author: Toru Kono, Advanced Surgery Center, 3-1, N 33, E 14, Higashi-ku, Sapporo Higashi Tokushukai Hospital, Sapporo, Hokkaido 065-0033, Japan, Tel: +81-11-722-1110; Fax: +81-11-722-0378; E-mail: kono@toru-kono.com

Received date: Dec 24, 2013, Accepted date: Feb 17, 2014, Published date: Feb 25, 2014

Copyright: (c) 2014 Kono T, et al. This is an open-access article distributed under the terms of the Creative Commons Attribution License, which permits unrestricted use, distribution, and reproduction in any medium, provided the original author and source are credited.

\begin{abstract}
In contrast to conventional single-target drugs, multi-component Kampo medicines are designed to achieve therapeutic effects through multiple drug targets. This article will discuss recent advances in mechanistic studies and the clinical effects of five representative Kampo formulations (hangeshashinto, daikenchuto, goshajinkigan, yokukansan and rikkunshito) for the treatment of adverse effects caused by anticancer drugs such as oral mucositis, diarrhea, neurotoxicity and abnormal behavior, for which western pharmaceuticals fail to adequately address.
\end{abstract}

Keywords: Kampo; Adverse effects; Chemotherapy; Mucositis; Diarrhea; Neurotoxicity

\section{Kampo Medicine and Western (Allopathic) Medicine: Integration or Segregation?}

Herbal medicines have been used by humans since antiquity and passed down for many generations until the present day as traditional medicines (e.g., Ayurveda, traditional Chinese medicine, Kampo). Among advanced countries, herbal medicine is a part of complementary and alternative medicine (CAM). It has been reported that almost $40 \%$ of adults in the United States use CAM therapy, but the most commonly used therapies are not covered by the health insurance like modern drugs [1]. By contrast, traditional Japanese herbal medicine called Kampo that evolved uniquely for more than 1500 years have been officially approved by the Japanese Ministry of Health, Labour and Welfare since 1976 as polyherbal extract formulations dispensed by physicians and pharmacists as prescription drugs. At present, over 130 Kampo formulae are covered by the Japanese national health insurance. Despite Kampo earning a respectable position in primary health care in Japan [2], the concept of herbal medicine continues to generate negative reactions among western trained physicians, both in Japan and abroad [1]. The reason behind such negative attitude is the lack of understanding about the fundamental differences between western and Kampo drugs. The first difference is that allopathic medicine has traditionally espoused the route of identifying a single component with a single molecular target to induce a specific therapeutic effect. Unfortunately, single component agents frequently act on other unintended targets, producing serious side effects. By contrast, Kampo contains multiple components that simultaneously act on multiple targets to produce complementary and synergistic therapeutic effects [3]. The second difference is the drug development process. Whereas the process of western drugs typically takes a minimum of 10 to 15 years of conducting pharmacokinetic and preclinical studies and placebocontrolled clinical trials before the drug is approved for the market, Kampo formulations have been empirically tested without placebo and refined for over 1000 to 1500 years [4]. Notwithstanding the long track record of clinical use in humans, insufficient framework for conducting large, well-designed preclinical and clinical studies has led to misconceptions among western trained physicians about the paucity of hard-nose evidence of Kampo. Fortunately, there has been a recent surge in scientifically robust basic and clinical studies on some of the Kampo medicines, which will be described in the following sections.

\section{The Role of Kampo in Oncology}

Despite the worldwide increase in the number of patients afflicted with colon cancer, it is one of the solid tumors that respond most favorably to chemotherapy; consequently chemotherapy has resulted in improving the quality of life and prolonging the survival rate for up to three years, even in patients with advanced cancer $[5,6]$. Yet the main caveat of chemotherapy is the inevitable side effects for which modern drugs fail to treat adequately, particularly conditions such as general malaise, anorexia, oral mucositis, and peripheral neuropathy. In addition, paralytic ileus after surgery for gastrointestinal cancer, chemotherapy-induced watery diarrhea, morphine-induced abnormal behavior in palliative care, and postoperative delirium remain as significant unmet clinical needs. The usefulness of Kampo for these symptoms that hinder the administration of full dosage of chemotherapy is increasingly recognized in Japan and the United States, and data from basic studies at the component level and reports on clinical evidence supporting Kampo's efficacy for these symptoms have exponentially increased as well. Although the amount of scientific data on Kampo still pales in comparison with those on western drugs, the highest level of scientific evidence (1a, meta-analysis of randomized controlled trials) has become available, paving way for the 
Citation: $\quad$ Kono T, Takeda H, Shimada M, Kase Y, Uezono Y (2014) Novel Therapeutics for Adverse Effects of Antitumor Therapy: The Promise of Multicomponent, Traditional Japanese Herbal Remedies. Carcinog \& Mutagen S8: 007. doi:10.4172/2157-2518.S8-007

Page 2 of 5

use of Kampo in oncology for conditions that often defy the best of modern drugs.

\section{Daikenchuto}

Daikenchuto is the most scientifically validated and frequently prescribed Kampo in Japan [1]. It contains Japanese pepper, processed ginger, and ginseng, all of which are dietary ingredients. Daikenchuto is primarily used for the treatment of postoperative paralytic ileus. Several studies have identified the pharmacologic mechanisms underlying the prokinetic effect of daikenchuto, including the promotion of neurotransmitters such as acetylcholine and calcitonin gene-related peptide (CGRP) [7-9]. Daikenchuto has also been reported to improve colonic blood flow via the stimulation of CGRP and adrenomedullin (ADM) release [1,10-13]. These two peptides share strikingly similar pharmacologic actions, including vasodilatory, anti-inflammatory, and antibacterial effects; however, they differ vastly in terms of their site of production, i.e., CGRP is produced by central and peripheral neuronal cells while ADM is synthesized by nonneuronal cells. When daikenchuto is administered directly into the intestinal lumen, intestinal epithelial cells enriched with ADM releases this peptide in large amounts, causing an immediate increase in blood flow in both the small and large intestine. This effect may appear to resemble that of the chili pepper, yet at the molecular level it differs greatly because the vasodilatory effect of daikenchuto is not inhibited by a selective antagonist of capsaicin.

With respect to the risk of aggravating inflammation by administering a vasodilatory agent like daikenchuto, our data from experimental colitis models have shown that daikenchuto normalizes blood flow in areas of poor circulation without affecting blood flow in areas of active inflammation. This is likely because CGRP and ADM receptors, the two molecular targets of daikenchuto, are occupied in inflammatory lesions and there are no more receptors left for daikenchuto to act on, which suggest that daikenchuto itself is not an analog of CGRP or ADM. When the effect of daikenchuto on ADM was probed further, we found that it induces ADM release from the intestinal epithelial cells in a dose- and time-dependent manner and that the active components of processed ginger (6-shogaol) and Japanese pepper (hydroxy- $\alpha$-sanshool) are primarily responsible for this action (Figure 1). Moreover, further investigation revealed that the two active components stimulate ADM release via activation of transient receptor potential channel ankyrin 1 (TRPA1) calcium channel in the intestinal epithelial cells of the small and large intestine. All of these effects are based on the assumption that the active components are absorbed. Pharmacokinetic studies conducted in Japan and the United States have demonstrated that the active components of processed ginger are indeed absorbed but ginseng components reach the colon intact without prior absorption, and that pharmacokinetics of daikenchuto are not affected sex and race $[14,15]$. The most intriguing finding was that Japanese pepper components are absorbed immediately upon administration.

With respect to the risk of daikenchuto exacerbating diarrhea, our data showed otherwise. Diarrhea can be broadly categorized into two types: nervous diarrhea and watery diarrhea. The former is thought to be caused by an overproduction of prokinetic neurotransmitters, but the neurotransmitters that daikenchuto targets are already mobilized in nervous diarrhea. Thus daikenchuto cannot possibly induce a pharmacologic effect in this type of diarrhea, and therefore, is highly unlikely to exacerbate nervous diarrhea. On the other hand, prostaglandin E2 (PGE2) is known as a key player in the pathogenesis of watery diarrhea. The active components of processed ginger such as shogaols and gingerols with multiple targets, including cyclooxygenase-2 (COX2) and arachidonic acid synthase (basic substances of PGE2), have been shown to inhibit PGE2 activity. In short, daikenchuto appears to be highly promising for the treatment of anticancer drug-induced watery diarrhea. In fact, many Japanese physicians proactively prescribe daikenchuto for intractable watery diarrhea.

Regarding clinical evidence, daikenchuto has been approved by the United States Food and Drug Administration as an investigational product (TU-100) and several clinical trials of TU-100 are currently underway (i.e., NCT01388933). The first placebo-controlled clinical trial in the United States was conducted at the prestigious Mayo Clinic in Rochester, which showed that TU-100 markedly improves gastrointestinal motility in healthy volunteers [16].

\section{Hangeshashinto}

Because of the high prevalence of oral mucositis among the general population irrespective of age and sex, patients who develop this condition as a side effect of anticancer treatment often fail to inform their physicians until the condition becomes severe[17,18]. In these patients, pain is often excruciating and severely restricts food intake in spite of normal appetite. Although oral mucositis heals naturally, it usually takes 10 days or longer. Considering that anticancer regimen is administered every week or once in two weeks, persistent mucositis is a huge burden on cancer patients. The pathogenic mechanisms of oral mucositis appear to involve chemotherapy-induced oxidative damage to mucosal cells and apoptosis induced by various cytokines, while pain arises from the upregulation of proinflammatory prostaglandins irritating the neurons. Hangeshashito contains processed ginger (6shogaol and 6-gingerol), a potent inhibitor of PGE2, as well as many other substances with this effect (Figure 1) [19,20]. For example, stimulation of human oral keratinocytes by IL- $1 \beta$ generates large amounts of PGE2. When hangeshashinto is added to this medium, it dose-dependently inhibits PGE2 and high doses produce an antiprostaglandin effect that is comparable to that of indomethacin. Besides 6-shogaol and 6-gingerol, active components of other hangeshashinto such as berberin (Scutellaria) and Coptisine (Coptis) have also been found to dose-dependently inhibit PGE2 via suppression of multiple critical enzymes like COX2 involved in the arachidonic acid metabolism (Figure 1) [20]. Further, studies using experimental oral mucositis model induced by anticancer drug fluorouracil (5-FU) revealed that hangeshashinto improves the rate of ulcer healing by two-fold by accelerating cell migration. Our results show that the pharmacologic mechanism of hangeshashinto is the inhibition of PGE2 synthesis which alleviates pain, allows for greater food intake, and promotes recovery of ulcers. In order to deliver hangeshashinto at the highest concentration possible to the damaged mucosa, we developed and tested the administration of hangeshashinto as an oral rinse. The first retrospective study of hangeshashinto oral rinse was highly promising [21]. The subsequent multicenter placebo-controlled, double-blind phase II study (HANGESHA-C study: UMIN000004287) showed that it can reduce ulcer healing time by half (from 10.5 days to 5.5 days) in patients with grade 2 or worse painful oral mucositis that requires intervention (unpublished data). 
Citation: $\quad$ Kono T, Takeda H, Shimada M, Kase Y, Uezono Y (2014) Novel Therapeutics for Adverse Effects of Antitumor Therapy: The Promise of Multicomponent, Traditional Japanese Herbal Remedies. Carcinog \& Mutagen S8: 007. doi:10.4172/2157-2518.S8-007

Page 3 of 5

\section{Goshajinkigan}

Oxaliplatin (L-OHP) is the most commonly used and highly effective anticancer agent for various gastrointestinal cancers. However, neurotoxicity develops in over $90 \%$ of patients and persists even after a year in $30 \%$ of these patients [22]. Oxaliplatin-based chemotherapy induced peripheral neuropathy worsens with cumulative oxaliplatin dose, and ranks among the most common dose- and choice-limiting as well as disabling side effect. Nevertheless, chemotherapy-induced peripheral neuropathy remains as a significant unmet clinical need.

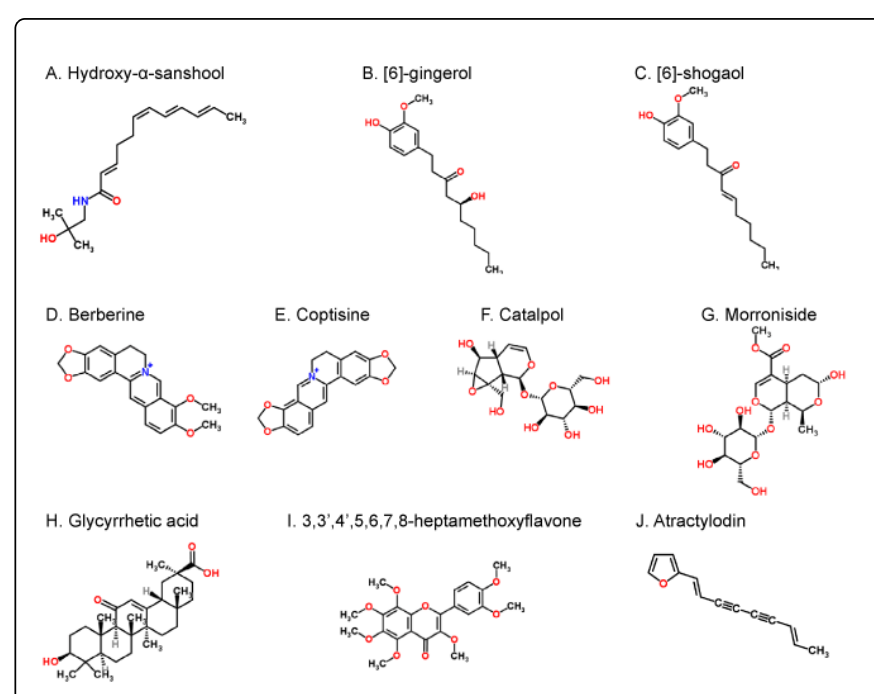

Figure 1: Active components of Kampo Component (Kampo). A. Hydroxy-a-sanshool (Daikenchuto), B. [6]-shogaol(Daikenchuto), C. [6]-Gingerol(Daikenchuto), D. Berberin (Hangeshashinto), E. Coptisine (Hangeshashinto), F. (Catalpol (Goshajinkigan), G. Morroniside (Goshajinkigan), H. Glycyrrhetic acid (Yokukansan), I. 3,3',4',5,6,7,8-heptamethoxyflavone (Rikkunshito) and J. Atractylodin (Rikkunshito). Structures created using ChemSpider, a free chemical structure database (http://www.chemspider.com/).

Goshajinkigan has been used for the treatment of chemotherapyinduced neurotoxicity on the basis of its mechanism of actions. These include nitric oxide-induced improvement in blood flow and analgesia via dynorphin and morphine receptor activation, and more recently, the inhibition of a certain TRP channel [23-25]. We first reported the effect of goshajinkigan against peripheral neuropathy in a retrospective study [24], and in a subsequent small-scale prospective study, we confirmed its efficacy [26]. In the following multicenter, placebo-controlled, double-blind, phase II study (GONE study: UMIN000002211), we found that goshajinkigan was efficacious in halting the onset of grade 3 or worse peripheral neuropathy [27]. Recently, we conducted a pharmacokinetic study in animals and discovered that the components (i.e., catalpol and morroniside) in goshajinkigan with neuroprotective and analgesic effects are indeed rapidly absorbed (unpublished data) (Figure 1). We anticipate that data from the ongoing investigation of pharmacokinetics of goshajinkigan will help inform clinicians to improve the method of its administration in the future.

\section{Yokukansan}

The highest level of scientific evidence (i.e., 1a, systematic review of randomized controlled trials) has been established for yokukansan among all the Kampo products available at this time. Hence yokukansan is quickly becoming the mainstay of treatment for symptoms associated with dementia, including abnormal behaviors. The excitatory glutamatergic system consists of inhibitory neurotransmitters that regulate excitation as well as reabsorption of excess glutamate by glutamate transporters in astrocytes (glial cells). Disturbance in these processes results in glutamate-induced neuronal hyperexcitability, leading to behavioral and psychological abnormalities such as delirium. Through restoring glutamate transporter dysfunction, yokukansan helps to lower glutamate levels and improve glutamate-mediated symptoms [28]. The active component with this effect is glycyrrhetic acid in Glycyrrhiza, the hydrolyzed form of glycyrrhizic acid by the intestinal bacteria (Figure 1) [29]. With a rise in the number of elderly patients undergoing cancer treatments, there has been a parallel increase in the incidence of abnormal behaviors triggered by the stress of surgery or chemotherapy in the absence of dementia. On the basis of mechanistic studies, yokukansan appears to be most effective when given prophylactically and symptomatically.

Up until now, antipsychotics have been primarily prescribed for the behavioral symptoms of dementia, but these often act on the dopaminergic and serotonergic systems [30-32] rather than the glutamatergic system, and consequently severely compromise the quality of life. By contrast, yokukansan is fast-acting without affecting the activities of daily living and its clinical effect in improving behavioral abnormalities associated with dementia has been confirmed in a meta-analysis of randomized controlled trials [33]. In addition, yokukansan shows early promise for the treatment of postoperative delirium as well as morphine-induced delirium in palliative care.

\section{Rikkunshito}

Cisplatin stimulates enterochromaffin cells distributed abundantly throughout the gastrointestinal tract to release serotonin, which in turn acts on 5-HT2B receptors in the stomach. Because 5-HT2B receptors inhibit ghrelin-producing cells, the production of the orexigenic hormone ghrelin is suppressed and anorexia develops $[34,35]$. The main component of Citrus Unshiu Peel in rikkunshito called 3,3', 4',5,6,7,8-heptamethoxyflavone (HMF) has been found to reverse ghrelin inhibition through selective antagonism of 5-HT2B receptors (Figure 1) [36]. In addition, atractylodin in Atractylodes has been identified to stimulate ghrelin/ghrelin receptor binding activity and signaling in the brain (Figure 1) [37].

In clinical trials, rikkunshito has been found to reverse decrease in plasma ghrelin level induced by chemotherapy such as cisplatin, and thereby improve symptoms such as nausea, vomiting, and anorexia $[34,38]$.

\section{Summary}

Each Kampo formula is like an orchestra in which the individual instruments (herbal components) are themselves magnificent players that have been carefully selected and whose combination has been time-tested over thousands of years to produce a musical masterpiece (treatment). Through the provision of Kampo, Japan's herbal products of unsurpassed quality and reliability, we hope to revitalize health care 
Citation: $\quad$ Kono T, Takeda H, Shimada M, Kase Y, Uezono Y (2014) Novel Therapeutics for Adverse Effects of Antitumor Therapy: The Promise of Multicomponent, Traditional Japanese Herbal Remedies. Carcinog \& Mutagen S8: 007. doi:10.4172/2157-2518.S8-007

Page 4 of 5

and contribute to improving the quality of life of patients around the world.

\section{References}

1. Kono T, Kanematsu T, Kitajima M (2009) Exodus of Kampo, traditional Japanese medicine, from the complementary and alternative medicines: is it time yet? Surgery 146: 837-840.

2. Motoo Y, Seki T, Tsutani K (2011) Traditional Japanese medicine, Kampo: its history and current status. Chin J Integr Med 17: 85-87.

3. Terasawa K (2004) Evidence-based Reconstruction of Kampo Medicine: Part-III-How Should Kampo be Evaluated? Evid Based Complement Alternat Med 1: 219-222.

4. Terasawa K (2004) Evidence-based Reconstruction of Kampo Medicine: Part I-Is Kampo CAM? Evid Based Complement Alternat Med 1: 11-16.

5. Denlinger CS, Engstrom PF (2011) Colorectal cancer survivorship: movement matters. Cancer Prev Res (Phila) 4: 502-511.

6. Hung A, Mullins CD (2013) Relative effectiveness and safety of chemotherapy in elderly and nonelderly patients with stage III colon cancer: a systematic review. Oncologist 18: 54-63.

7. Jin XL, Shibata C, Naito H, Ueno T, Funayama Y, et al. (2001) Intraduodenal and intrajejunal administration of the herbal medicine, dai-kenchu-tou, stimulates small intestinal motility via cholinergic receptors in conscious dogs. Dig Dis Sci 46: 1171-1176.

8. Nagano T, Itoh H, Takeyama M (1999) Effect of Dai-kenchu-to on levels of 3 brain-gut peptides (motilin, gastrin and somatostatin) in human plasma. Biol Pharm Bull 22: 1131-1133.

9. Shibata C, Sasaki I, Naito H, Ueno T, Matsuno S (1999) The herbal medicine Dai-Kenchu-Tou stimulates upper gut motility through cholinergic and 5-hydroxytryptamine 3 receptors in conscious dogs. Surgery 126: 918-924.

10. Kaneko A, Kono T, Miura N, Tsuchiya N, Yamamoto M (2013) Preventive Effect of TU-100 on a Type-2 Model of Colitis in Mice: Possible Involvement of Enhancing Adrenomedullin in Intestinal Epithelial Cells. Gastroenterol Res Pract 2013: 384057.

11. Kono T, Kaneko A, Omiya Y, Ohbuchi K, Ohno N, et al. (2013) Epithelial transient receptor potential ankyrin 1 (TRPA1)-dependent adrenomedullin upregulates blood flow in rat small intestine. Am J Physiol Gastrointest Liver Physiol 304:G428-436.

12. Kono T, Koseki T, Chiba S, Ebisawa Y, Chisato N, et al. (2008) Colonic vascular conductance increased by Daikenchuto via calcitonin generelated peptide and receptor-activity modifying protein 1 . J Surg Res 150 : 78-84.

13. Kono T, Omiya Y, Hira Y, Kaneko A, Chiba S, et al. (2011) Daikenchuto (TU-100) ameliorates colon microvascular dysfunction via endogenous adrenomedullin in Crohn's disease rat model. J Gastroenterol 46: 1187-1196.

14. Munekage $M$, Ichikawa $\mathrm{K}$, Kitagawa $\mathrm{H}$, Ishihara $\mathrm{K}$, Uehara $\mathrm{H}$, et al. (2013) Population pharmacokinetic analysis of daikenchuto, a traditional Japanese medicine (Kampo) in Japanese and US health volunteers. Drug Metab Dispos 41:1256-1263.

15. Munekage M, Kitagawa H, Ichikawa K, Watanabe J, Aoki K, et al. (2011) Pharmacokinetics of daikenchuto, a traditional Japanese medicine (kampo) after single oral administration to healthy Japanese volunteers. Drug Metab Dispos 39: 1784-1788.

16. Manabe N, Camilleri M, Rao A, Wong BS, Burton D, et al. (2010) Effect of daikenchuto (TU-100) on gastrointestinal and colonic transit in humans. Am J Physiol Gastrointest Liver Physiol 298: G970-975.

17. Quinn B (2013) Efficacy of a supersaturated calcium phosphate oral rinse for the prevention and treatment of oral mucositis in patients receiving high-dose cancer therapy: a review of current data. Eur J Cancer Care (Engl) 22: 564-579.

18. Stindt D, Brown MJ (2013) Chemotherapy-Induced mucositis. Adv NPs PAs 4: 27-28.
19. Kase Y, Hayakawa T, Aburada M, Komatsu Y, Kamataki T. (1997) Preventive effects of Hange-shashin-to on irinotecan hydrochloridecaused diarrhea and its relevance to the colonic prostaglandin E2 and water absorption in the rat. Jpn J Pharmacol 75:407-413.

20. Kono T, Kaneko A, Matsumoto C, Miyagi C, Ohbuchi K, et al. (2014) Multitargeted Effects of Hangeshashinto for Treatment of Chemotherapy-Induced Oral Mucositis on Inducible Prostaglandin E2 Production in Human Oral Keratinocytes. Integr Cancer Ther.

21. Kono T, Satomi M, Chisato N, Ebisawa Y, Suno M, et al. (2010) Topical Application of Hangeshashinto (TJ-14) in the Treatment of Chemotherapy-Induced Oral Mucositis. World J Oncol 1:232-235.

22. Andre T, Boni C, Mounedji-Boudiaf L, Navarro M, Tabernero J, et al. (2004) Oxaliplatin, fluorouracil, and leucovorin as adjuvant treatment for colon cancer. N Engl J Med 350:2343-2351.

23. Hidaka T, Shima T, Nagira K, Ieki M, Nakamura T, et al. (2009) Herbal medicine Shakuyaku-kanzo-to reduces paclitaxel-induced painful peripheral neuropathy in mice. Eur J Pain 13: 22-27.

24. Kono T, Mamiya N, Chisato N, Ebisawa Y, Yamazaki H, et al. (2011) Efficacy of goshajinkigan for peripheral neurotoxicity of oxaliplatin in patients with advanced or recurrent colorectal cancer. Evid Based Complement Alternat Med 2011: 418481.

25. Yoshida T, Sawa T, Ishiguro T, Horiba A, Minatoguchi S, et al. (2009) The efficacy of prophylactic Shakuyaku-Kanzo-to for myalgia and arthralgia following carboplatin and paclitaxel combination chemotherapy for non-small cell lung cancer. Support Care Cancer 17: 315-320.

26. Nishioka M, Shimada M, Kurita N, Iwata T, Morimoto S, et al. (2011) The Kampo medicine, Goshajinkigan, prevents neuropathy in patients treated by FOLFOX regimen. Int J Clin Oncol 16: 322-327.

27. Kono T, Hata T, Morita S, Munemoto Y, Matsui T, et al. (2013) Goshajinkigan oxaliplatin neurotoxicity evaluation (GONE): a phase 2, multicenter, randomized, double-blind, placebo-controlled trial of goshajinkigan to prevent oxaliplatin-induced neuropathy. Cancer Chemother Pharmacol.

28. Kawakami Z, Kanno H, Ueki T, Terawaki K, Tabuchi M, et al. (2009) Neuroprotective effects of yokukansan, a traditional Japanese medicine, on glutamate-mediated excitotoxicity in cultured cells. Neuroscience 159:1397-1407.

29. Kawakami Z, Ikarashi Y, Kase Y. (2010) Glycyrrhizin and its metabolite 18 beta-glycyrrhetinic acid in glycyrrhiza, a constituent herb of yokukansan, ameliorate thiamine deficiency-induced dysfunction of glutamate transport in cultured rat cortical astrocytes. Eur J Pharmacol 626:154-158.

30. Mizoguchi K, Shoji H, Tanaka Y, Tabira T (2011) Ameliorative effect of traditional Japanese medicine yokukansan on age-related impairments of working memory and reversal learning in rats. Neuroscience 177: 127-137.

31. Mizoguchi K, Tanaka Y, Tabira T (2010) Anxiolytic effect of a herbal medicine, yokukansan, in aged rats: involvement of serotonergic and dopaminergic transmissions in the prefrontal cortex. J Ethnopharmacol 127: 70-76.

32. Nishi A, Yamaguchi T, Sekiguchi K, Imamura S, Tabuchi M, et al. (2012) Geissoschizine methyl ether, an alkaloid in Uncaria hook, is a potent serotonin â,A receptor agonist and candidate for amelioration of aggressiveness and sociality by yokukansan. Neuroscience 207: 124-136.

33. Matsuda Y, Kishi T, Shibayama H, Iwata N (2013) Yokukansan in the treatment of behavioral and psychological symptoms of dementia: a systematic review and meta-analysis of randomized controlled trials. Hum Psychopharmacol 28: 80-86.

34. Ohno T, Yanai M, Ando H, Toyomasu Y, Ogawa A, et al. (2011) Rikkunshito, a traditional Japanese medicine, suppresses cisplatininduced anorexia in humans. Clin Exp Gastroenterol 4: 291-296.

35. Yakabi K, Sadakane C, Noguchi M, Ohno S, Ro S, et al. (2010) Reduced ghrelin secretion in the hypothalamus of rats due to cisplatin-induced anorexia. Endocrinology 151: 3773-3782. 
Citation: Kono T, Takeda H, Shimada M, Kase Y, Uezono Y (2014) Novel Therapeutics for Adverse Effects of Antitumor Therapy: The Promise of Multicomponent, Traditional Japanese Herbal Remedies. Carcinog \& Mutagen S8: 007. doi:10.4172/2157-2518.S8-007

Page 5 of 5

36. Takeda H, Sadakane C, Hattori T, Katsurada T, Ohkawara T, et al. (2008) Rikkunshito, an herbal medicine, suppresses cisplatin-induced anorexia in rats via 5-HT2 receptor antagonism. Gastroenterology 134: 2004-2013.

37. Fujitsuka N, Asakawa A, Uezono Y, Minami K, Yamaguchi T, et al. (2011) Potentiation of ghrelin signaling attenuates cancer anorexiacachexia and prolongs survival. Transl Psychiatry 1: e23.
38. Seike J, Sawada T, Kawakita N, Yamamoto Y, Yuasa Y, et al. (2011) A New Candidate Supporting Drug, Rikkunshito, for the QOL in Advanced Esophageal Cancer Patients with Chemotherapy Using Docetaxel/5-FU/ CDDP. Int J Surg Oncol. 